\title{
Report for the Office of Scientific and Technical Information: Population Modeling of the Emergence and Development of Scientific Fields
}

\author{
Luis M. A. Bettencourt \\ Theoretical Division, T-7 \\ Los Alamos National Laboratory
}

\author{
Carlos Castillo-Chávez \\ Department of Mathematics and Statistics \\ Arizona State University
}

\section{David Kaiser}

Program in Science, Technology, and Society, and Department of Physics

Massachusetts Institute of Technology

David E. Wojick

Office of Scientific and Technical Information

US Department of Energy

October 4, 2006

\section{Introduction}

The accelerated development of digital libraries and archives, in tandem with efficient search engines and the computational ability to retrieve and parse massive amounts of information, are making it possible to quantify the time evolution of scientific literatures. These data are but one piece of the tangible recorded evidence of the processes whereby scientists create and exchange information in their journeys towards the generation of knowledge. As such, these tools provide a proxy with which to study our ability to innovate.

Innovation has often been linked with prosperity and growth and, consequently, trying to understand what drives scientific innovation is of extreme interest. Identifying sets of population characteristics, factors, and mechanisms that enable scientific communities to remain at the cutting edge, accelerate their growth, or increase their ability to re-organize around new themes or research topics is therefore of special significance. Yet generating a quantitative understanding of the factors that make scientific fields arise and/or become more or less productive is still in its infancy. This is precisely the type of knowledge most needed for promoting and sustaining innovation. Ideally, the efficient and strategic allocation of resources on the part of funding agencies and corporations would be driven primarily by knowledge of this type. 
Early steps have been taken toward such a quantitative understanding of scientific innovation. Some have focused on characterizing the broad properties of relevant time series, such as numbers of publications and authors in a given field (see $[1,2,3]$, and $[4]$ and references therein). Others have focused on the structure and evolution of networks of co-authorship and citation, see e. g. [5, 6, 7]. Together these types of studies provide muchneeded statistical analyses of the structure and evolution of scientific communities. Despite these efforts, however, crucial elements of prediction have remained elusive. Building on many of these earlier insights, we provide here a coarse-grained approach to modeling the time-evolution of scientific fields mathematically, through adaptive models of contagion. That is, our models are inspired by epidemic contact processes, but take into account the social interactions and processes whereby scientific ideas spread - social interactions gleaned from close empirical study of historical cases [4]. Variations in model parameters can increase or hamper the speed at which a field develops. In this way, models for the spread of "infectious" ideas can be used to identify pressure points in the process of innovation that may allow for the evaluation of possible interventions by those responsible for promoting innovation, such as funding agencies.

This report is organized as follows: Section 2 introduces and discusses the population model used here to describe the dynamics behind the establishment of scientific fields. The approach is based on a succinct (coarse) description of contact processes between scientists, and is a simplified version of a general class of models developed in the course of this work. We selected this model based primarily on its ability to treat a wide range of data patterns efficiently, across several different scientific fields. We also describe our methods for estimating parameter values, our optimization techniques used to match the model to data, and our method of generating error estimates. Section 3 presents brief accounts of six case studies of scientific evolution, measured by the growth in number of active authors over time, and shows the results of fitting our model to these data, including extrapolations to the near future. Section 4 discusses these results and provides some perspectives on the values and limitations of the models used. We also discuss topics for further research which should improve our ability to predict (and perhaps influence) the course of future scientific research. Section 5 provides more detail on the broad class of epidemic models developed as part of this project.

\section{Models and Parameter Estimation}

Our starting point is a generalized SEIR (Susceptible, Exposed, Infected, Recovered) epidemic model. Such models include terms familiar from epidemiological studies, such as contact rates between individuals and latency and recovery times. The model also includes (exponential) population growth and multiple contacts between members of the exposed and infected classes, terms that proved important in our previous study of the dynamics of how Feynman diagrams spread [4]. The model is 
written explicitly as

$$
\begin{aligned}
\frac{d S}{d t} & =\Lambda N-\beta S \frac{I}{N} \\
\frac{d E}{d t} & =\beta S \frac{I}{N}-\kappa E-\rho E \frac{I}{N}, \\
\frac{d I}{d t} & =\kappa E+\rho E \frac{I}{N}-\gamma I, \\
\frac{d R}{d t} & =\gamma I,
\end{aligned}
$$

where $S(t)$ is the size of the susceptible population at time $t, E(t)$ is the size of the exposed class, $I(t)$ is the size of the infected class (that is, those who have adopted the new scientific idea), and $R(t)$ is the size of the population who have recovered (no longer publishing on the topic). We shall refer to the size of the entire population, the sum over these classes, as $N$. Note that we did not include an exit (or death) term, as this tends to be very small, and is 'subsumed' by the recovered class. In this model, the population, $N$, grows exponentially with rate $\Lambda$. In some instances, indicated explicitly below, the growth term will be written as $\Lambda$, instead of $\Lambda N$, and may not apply to the entire duration of the dynamics.

The remaining parameters account for the probability and effectiveness of a contact with an adopter $\beta$, the standard latency time $1 / \kappa$, the duration of the infectious period $1 / \gamma$, and the probability that an exposed person has multiple effective contacts with other adopters, $\rho$. The model may be visualized as in Fig. 1 . The reproductive number for the model - that is, the average number of new people infected by a given infected individual - is $R_{0}=\beta / \gamma$. This is a simplified version of a more general family of models developed in the course of this work, which feature multiple latency classes (see Section 5, below, and also [8]).

Parameter estimation is performed via a stochastic ensemble algorithm as described in [4]. The parameters describing the initial conditions $\left[S\left(t_{0}\right), E\left(t_{0}\right), I\left(t_{0}\right), R\left(t_{0}\right)\right]$ and the dynamical parameters are organized as a vector of real-valued numbers. An ensemble of such vectors or strings is generated via the perturbation of a "progenitor" string. The fitness of the resulting strings - referred to collectively as a "generation," given the similarity to genetic algorithms - is evaluated by comparison with the data. The 'best' strings are chosen to spawn the subsequent generation, and so on, until the procedure converges, that is, the fitness ceases to improve. Several checks are performed to guarantee that the absolute (global) best fit was reached. The procedure generates not only a best fit solution, showing the smallest deviation to the data, but also an ensemble of good strings, from which uncertainty in the solution is quantified.

Below we apply this stochastic ensemble optimization procedure to data on the number of authors participating in the advent and subsequent growth of several fields, in the aftermath of a discovery, breakthrough, or surge of interest. 


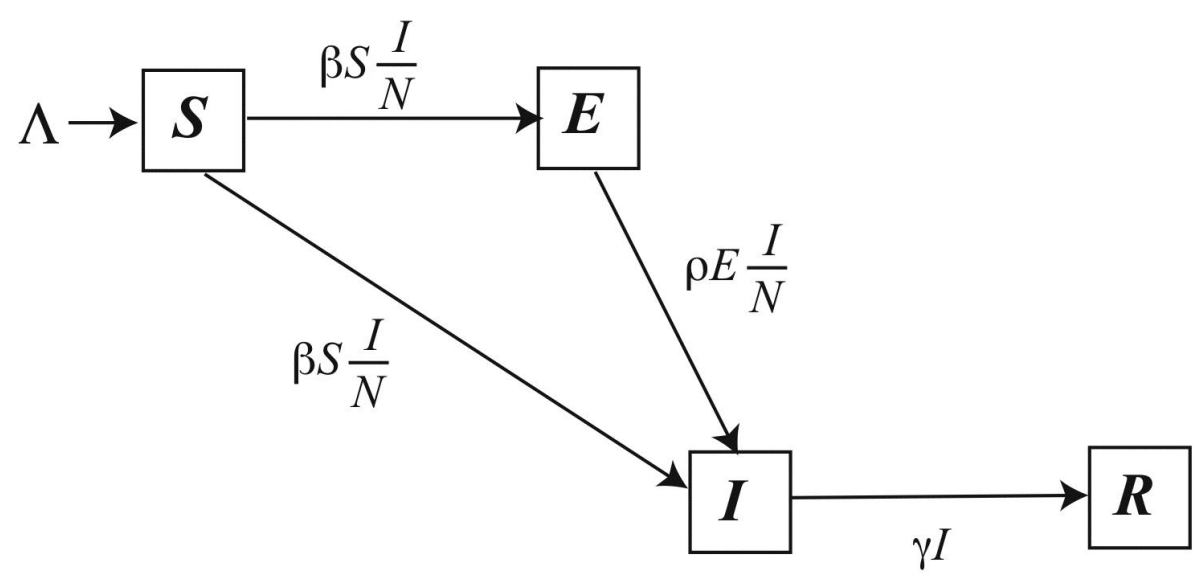

Figure 1: Transfer diagram for the SEIR model.

\section{Case studies and population modeling results}

In this section we present several studies of the emergence of scientific fields and results of modeling the evolution in numbers of authors using the models introduced in section 2. Parameter estimates are given at the end of the section for all cases, and discussion, conclusions and outlook are presented in the next section.

\section{Cosmological Inflation}

Cosmological inflation is a theory, proposed by A. Guth and A. Linde in 1981, describing the exponential expansion of the early universe. Remarkably, it provides solutions to most open issues that arise when combining big bang cosmology with astrophysical observations. Originally it was conceived to solve the problem of overproduction of cosmological defects (mostly monopoles, see cosmic strings below), but perhaps its strongest feature is to provide a prediction for the initial energy density perturbations necessary to seed the large-scale structure of the universe (see [9]).

The data shown here result from literature searches based on citations to an early set of publications in the field as well as to later review articles. The time evolution of the field, measured in terms of numbers of authors, is peculiar when compared to other case studies discussed below: it is approximately linear, appearing least like a classic epidemic curve. Nevertheless, our SEIR model with population growth and large contact rate describes the data very well. (See Fig. 2.)

\section{Cosmic Strings}




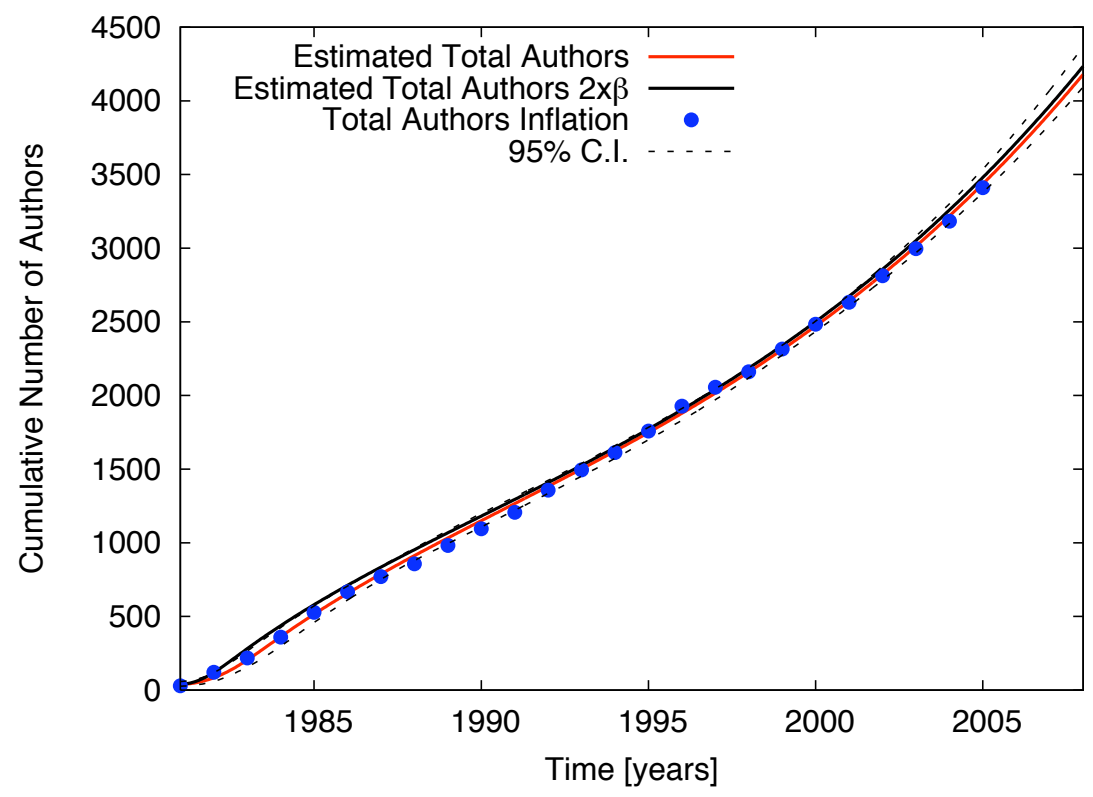

Figure 2: The temporal evolution of the cumulative number of authors publishing in Cosmological Inflation (blue dots), the fit from the model (red line), and 95\% confidence interval (dashed lines). The solid black line shows the best-fit solution with twice the contact rate between susceptible and adopters. The small sensitivity of the solution to changes in the contact rate is the result of the solution placing the $E$ and $I$ classes at their fixed points, slowly driven by an increasing population $N$. 
Cosmic strings and other topological defects are non-perturbative solutions of unified theories of elementary interactions that may have been formed in phase transitions in the early universe. In 1976 T. W. B. Kibble suggested that the internal symmetry groups of modern theories of particle physicstaken together with the Higgs mechanism, necessary to render elementary particles massivelead inexorably to phase transitions in the early universe, similar to, but generally more complex than those in superconductors and certain superfluids. It follows that, in the early universe, as in these materials, topological defects could form that would be stable and able to concentrate vast amounts of energy in their profiles, be they monopoles, cosmic strings, or domain walls. These defects could seed the large-scale structure of the universe by providing energy (and momentum) inhomogeneities upon which baryonic matter could fall.

For some time, until the mid-1990s, cosmic strings and cosmological inflation were rival theories contending to explain the features of the observed universe. More recent precise measurements of the cosmic microwave background and type Ia supernovae, however, seem to weigh more strongly in favor of inflation, making the case for cosmological defects increasingly constrained [9]. This most likely accounts for the up-turn in numbers of authors publishing on inflation and the declining rate of new authors pursuing cosmic strings. (See Fig. 3.)

\section{Prions}

Prions (proteinaceous infectious particle) are abnormally configured proteins, which were shown in 1982 , by S. B. Prusiner and colleagues, to cause scrapie, a transmissible spongiform encephalopathy in sheep. It was later recognized that other related diseases, such as Kuru (Kreuzfeld Jacobs disease in humans) and BSE (mad cow disease) are also caused by prions, and not by a virus or any other conventional infectious agent. The discovery was followed, a decade later, by great public health scares, principally associated with BSE in the UK, which contributed to raise the profile of the field. By the late 90s research in prions had become underfunded, and the field started to show signs of slow down. For the discovery of prions and their connection to spongiform encephalopathies Stanley B. Prusiner won the Nobel prize in Medicine in 1997 (see http://nobelprize.org/nobel_prizes/medicine/laureates/1997/press.html).

The data used here were obtained from a keyword search for prion among scientific publications (having eliminated a genus of like named birds), and also includes research in scrapie. (See Fig. 4.) In the near future we intend to enlarge this dataset by including scientific literatures dealing with Kuru, BSE, and related nervous system pathologies.

\section{H5N1 Influenza}

H5N1 influenza is a subtype of the influenza A virus that causes "bird flu." It is presently 


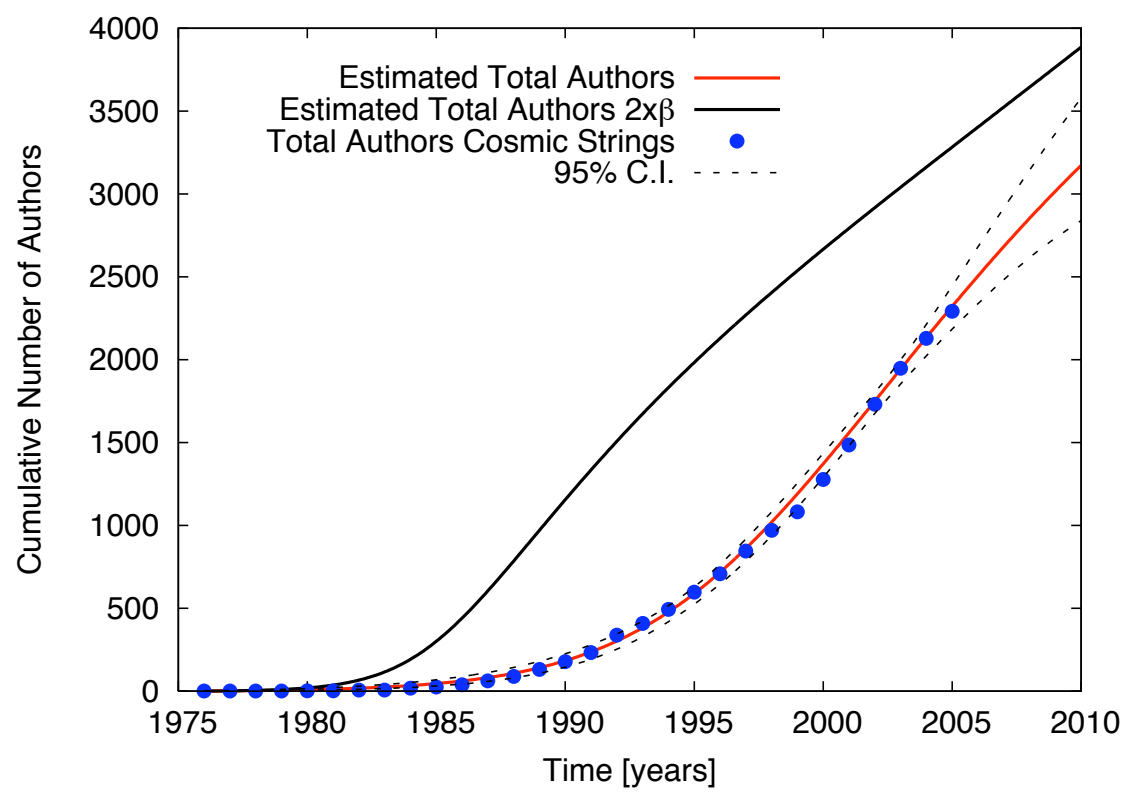

Figure 3: The temporal evolution of the cumulative number of authors publishing in Cosmic Strings (blue dots), the fit from the model (red line), and 95\% confidence interval (dashed lines). The solid black line shows the best-fit solution with twice the contact rate between susceptible and adopters. 


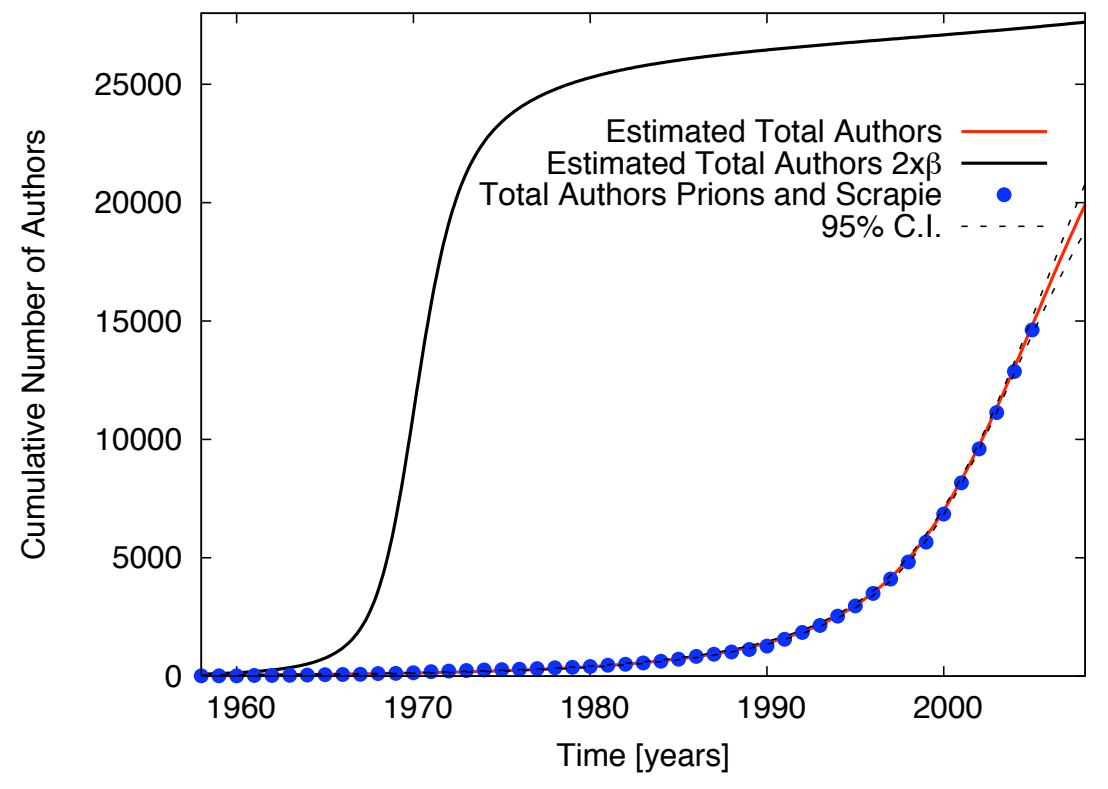

Figure 4: The temporal evolution of the cumulative number of authors publishing in Prions and Scrapie (blue dots), the fit from the model (red line), and 95\% confidence interval (dashed lines). The solid black line shows the best-fit solution with twice the contact rate between susceptible and adopters. 
a disease of birds, but there have been over 200 confirmed cases of human infection, mostly in Southeast Asia, with an observed case mortality rate of about $57 \%$. For these reasons H5N1 influenza is thought to have the potential to cause the next great human influenza pandemic. As a result research in $\mathrm{H} 5 \mathrm{~N} 1$ and other types of influenza has gained extraordinary impetus over the last few years. Research on the H5N1 subtype started in earnest after 1997, when the first human cases of the disease were identified in Hong Kong. New, larger outbreaks in the last 2-3 years have lead to great scientific and public interest in the field and its relation to other influenza types. Its literature spans themes in health policy, epidemiology and molecular biology. (See Fig. 5.)

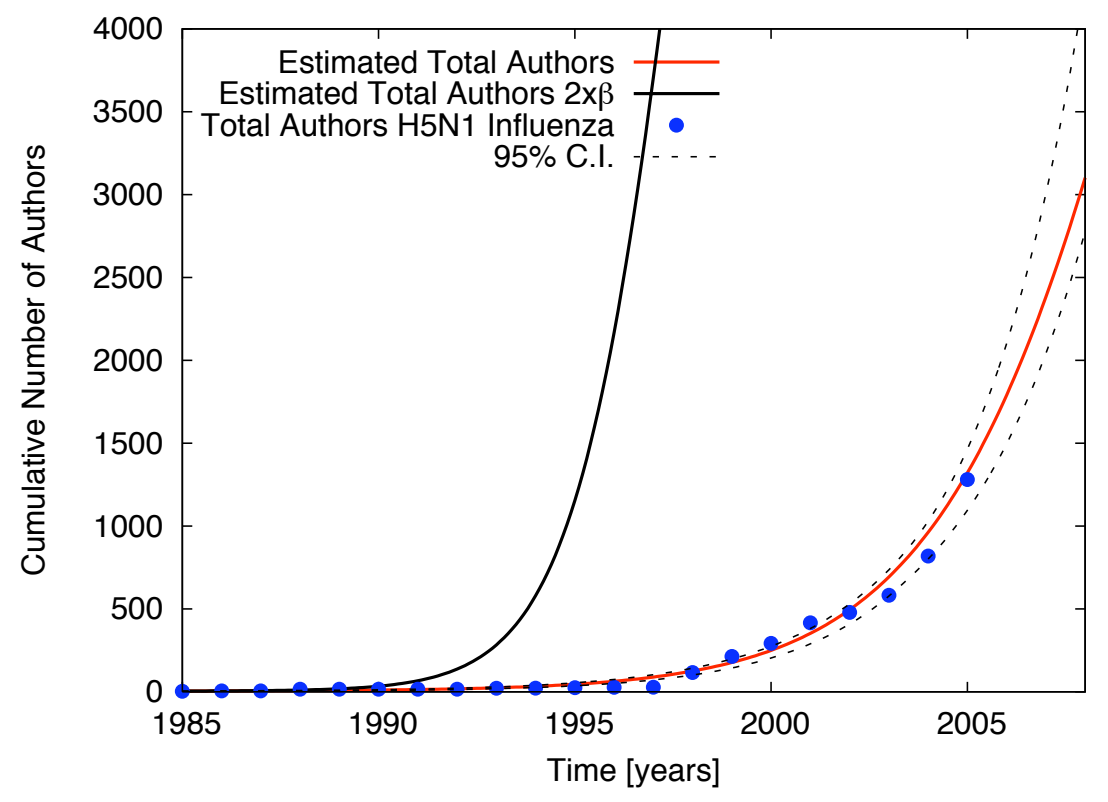

Figure 5: The temporal evolution of the cumulative number of authors publishing in H5N1 influenza (blue dots), the fit from the model (red line), and 95\% confidence interval (dashed lines). The solid black line shows the best-fit solution with twice the contact rate between susceptible and adopters.

\section{Carbon Nanotubes}

Carbon nanotubes are a recently discovered allotrope of carbon, which promises to generate a whole family of new materials and potentially revolutionize nano-engineering. 
Research in this area started in 1991, when Sumio Iijima of NEC in Japan discovered a new method (arc discharge) to produce them, although nanotubes had been described before in the literature. It is hoped that these materials may usher in many promising engineering solutions at the nanoscale due to their enormous strength, lightness, and conductive properties of heat and electrical currents.

We built a database of scientific publications in the field by performing a keyword search for "carbon nanotubes" among scientific publications, and excluding "tubules," which is a term that tends to co-occur with carbon, but refers instead to cells' skeletons. (See Fig. 6.)

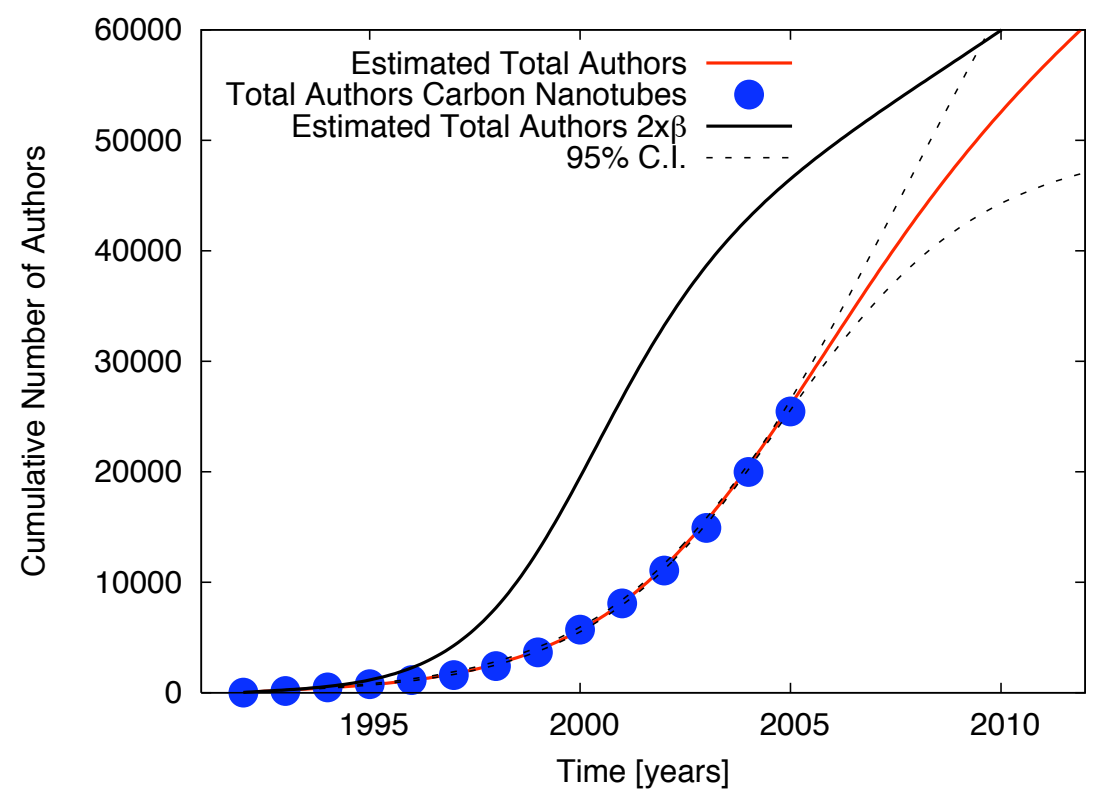

Figure 6: The temporal evolution of the cumulative number of authors publishing in Carbon Nanotubes (blue dots), the fit from the model (red line), and 95\% confidence interval (dashed lines). The solid black line shows the best-fit solution with twice the contact rate between susceptible and adopters.

\section{Quantum Computing and Computation}

Quantum Computing is an emerging field of research dedicated to the discovery of new devices and theoretical implementations of states that are genuinely quantum-mechanical and can be manipulated for computation. A quantum computer would be able to perform 
certain operations (such as factorization) much faster and solve physical quantum models more naturally and efficiently than any classical computer. The field is naturally multidisciplinary involving research in quantum theory, material science, and engineering. It dates back to the late 1960s and 1970s, but gained momentum with several theoretical breakthroughs in the 1980s and 1990s. In 1982 Richard Feynman showed how quantum systems could be used to do computation. In 1985 David Deutch proved that any physical process can, in principle, be modeled perfectly in a quantum computer. In the 1990s algorithms by Shor and Grover and NMR experiments by Laflamme et al. demonstrated explicitly the advantage of quantum algorithms in certain computational tasks and the first implementations of the technology. Today the field remains very active, especially in finding hardware implementations that can both be resistant to quantum decoherence and amenable to external control. (See Fig. 7.)

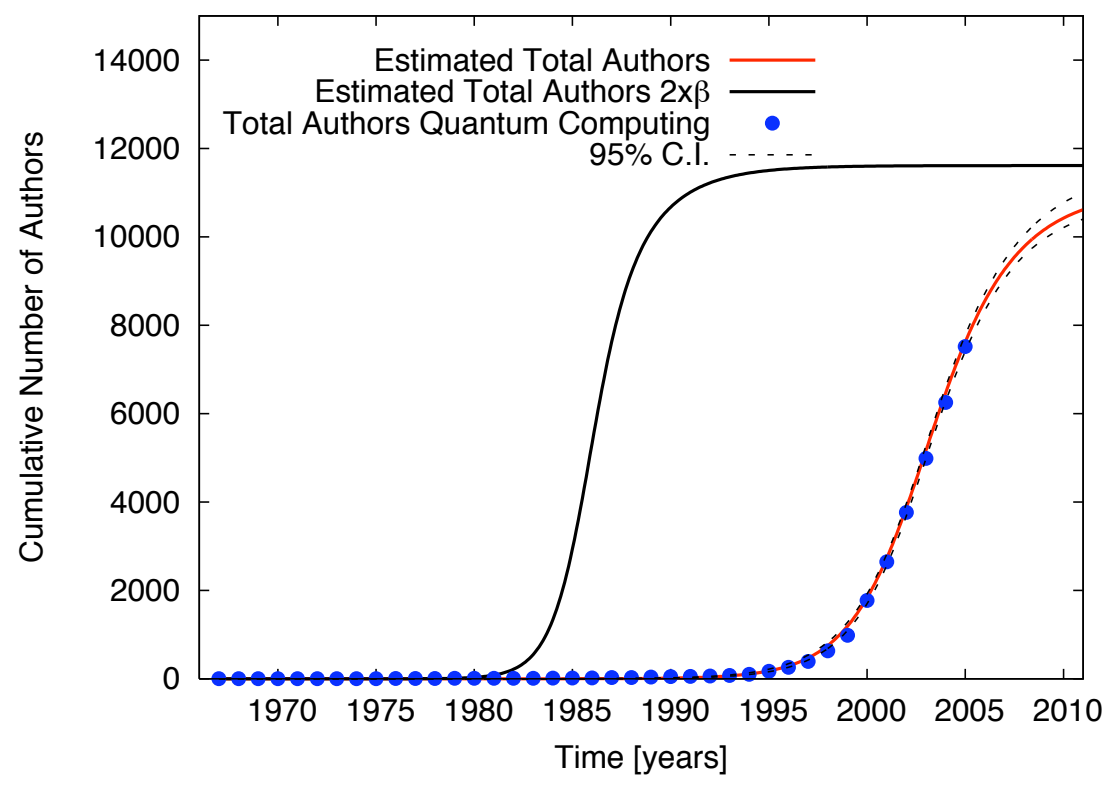

Figure 7: The temporal evolution of the cumulative number of authors publishing in Quantum Computing and Computation (blue dots), the fit from the model (red line), and 95\% confidence interval (dashed lines). The solid black line shows the best-fit solution with twice the contact rate between susceptible and adopters. 


\begin{tabular}{|c||c|c|c|c|c|c|c|c|c||c|}
\hline Parameters & $\mathrm{S}\left(\mathrm{t}_{\mathrm{p}}\right)$ & $\mathrm{E}\left(\mathrm{t}_{\mathrm{p}}\right)$ & $\mathrm{I}\left(\mathrm{t}_{\mathrm{p}}\right)$ & $\mathrm{R}\left(\mathrm{t}_{\mathrm{p}}\right)$ & $\beta$ & $\Lambda$ & $\mathrm{K}$ & $\rho$ & $\gamma$ & $R_{0}$ \\
\hline $\begin{array}{c}\text { Cosmological } \\
\text { Inflation }\end{array}$ & $930 \pm 1$ & 6 & $37 \pm 1$ & 2 & $13.41 \pm 0.28$ & 0.07 & 0.20 & 0 & 0.21 & $64.6 \pm 1.5$ \\
\hline $\begin{array}{c}\text { Cosmic } \\
\text { Strings }\end{array}$ & $14 \pm 9$ & 5 & 0 & 0 & $4.45 \pm 0.42$ & $159.1 \pm 2.7 *$ & $0.25 \pm 0.02$ & 0 & $1.73 \pm 0.19$ & $2.58 \pm 0.11$ \\
\hline $\begin{array}{c}\text { Prions } \\
\text { \& Scrapie }\end{array}$ & $14262 \pm 1368$ & 1 & $8 \pm 1$ & $7 \pm 2$ & $0.69 \pm 0.05$ & $469 \pm 25^{*}$ & $0.22 \pm 0.01$ & $18.4 \pm 1.24$ & $0.37 \pm 0.03$ & $1.87 \pm 0.03$ \\
\hline $\begin{array}{c}\text { H5N1 } \\
\text { Influenza }\end{array}$ & $9057 \pm 200$ & 1 & 0 & 0 & $1.47 \pm 0.02$ & $138 \pm 10^{*}$ & $0.71 \pm 0.01$ & 0 & $0.6 \pm 0.01$ & $2.44 \pm 0.03$ \\
\hline $\begin{array}{c}\text { Carbon } \\
\text { Nanotubes }\end{array}$ & $30464 \pm 5976$ & $501 \pm 24$ & 1 & 1 & $0.99 \pm 0.05$ & $0.04 \pm 0.01$ & $0.50 \pm 0.03$ & $0.03 \pm 0.06$ & $0.10 \pm 0.05$ & $9.72 \pm 1.71$ \\
\hline $\begin{array}{c}\text { Quantum } \\
\text { Computing }\end{array}$ & $11627 \pm 91$ & 0 & 0 & 0 & $3.78 \pm 0.09$ & $1.03 \pm 0.02 * *$ & $0.41 \pm 0.02$ & $0.77 \pm 0.03$ & $1.18 \pm 0.02$ & $3.20 \pm 0.11$ \\
\hline
\end{tabular}

* Indicates a linear growth term $\Lambda$, not $\Lambda N$ in the equations for $S$.

** Susceptible population growth starts in 1990.

Table 1: Parameter estimates for the models of Section 2, and data sets described in Section 3. Best fit curves and $95 \%$ Confidence Intervals for the model estimates are shown in Figs. $2-7$.

\section{Discussion and Outlook}

The case studies developed here show that population models analogous to those of epidemiology, suitably adapted, provide excellent bases with which to describe quantitatively the emergence and development of scientific fields across the natural sciences. Remarkably, our simple model describes equally well theoretical fields (such as cosmic strings and inflation) as experimental ones (such as prions and carbon nanotubes), or those that include both kinds of activity (such as quantum computing and H5N1 influenza). This in itself is an important demonstration that the type of model we devised to treat one particular case in great detail (the spread of Feynman diagrams among theoretical physicists) [4] may be applied much more broadly, with equally impressive fits to empirical data.

Moreover, the parameter estimates for these many cases reveal several features that make intuitive sense. For example, the two cases that draw authors from only one narrowly defined specialty - inflation and cosmic strings - show the smallest initial populations of susceptibles, $S\left(t_{0}\right)$, whereas those fields that cross disciplinary boundaries, potentially attracting researchers from many different scientific areas, reveal correspondingly larger initial populations of susceptibles. Likewise, the two purely theoretical fields (again, inflation and cosmic strings) show the greatest effectiveness of contact, $\beta$; those fields which most thoroughly mix theoretical and experimental components (quantum computing and H5N1 influenza) have intermediate values for $\beta$; while the more purely experimental fields (prions and carbon nanotubes) have the smallest values for $\beta$. As one might expect, it is one thing to practice and master a pencil-and-paper technique; quite another to build and 
oversee an entire working lab group.

We must note however that although the fundamental dynamics of contact and spread may be analogous between the spread of ideas and disease, many characteristics of the two dynamics are fundamentally different. First, the nature of the contacts is clearly distinct. Many scientific contacts are prolonged and based on mentor/apprenticeship relationships such as those between advisors and students or postdocs. This fact also highlights that recruitment plays an important role, alongside conversion of susceptibles in the growth of a field. Parameter estimation supports these expectations, showing large numbers of initial susceptibles and/or population growth typically of a few percent a year.

Compared to most diseases, scientific ideas spread slowly, taking years to become adopted by a significant number of practitioners. They also show substantial contact rates over these time scales, perhaps the result of the many intentional social structures - $\mathrm{PhD}$ programs, postdocs, meetings, workshops, etc. - designed to foster sustained interactions. The result is that although incubation times range between 1.4-5 years and infectious periods between 6 months and 10 years, all cases show large basic reproductive numbers $R_{0}$ between 1.8 and 64 . This point seems to be very general and a manifestly different feature between ideas and infectious diseases: useful ideas may never be forgotten, leading to very long infectious periods and therefore large $R_{0}$.

It is also typical of the spread of ideas that long and repeated contacts between adopters and susceptible individuals take place in order for the concept or technique to be transmitted. Here we modeled these processes via a contact term between exposed and infected, proportional to the contact rate $\rho$. In most of our case studies, however, with the exception of Prions \& Scrapie, estimation of this term shows very small values for $\rho$, indicating that perhaps persistent contacts were not essential, or that a different modeling strategy might be necessary to capture such effects.

While most case studies showed growth dynamics that are familiar from other invasion processes, two of our examples were peculiarly different. First, Cosmological Inflation shows growth in numbers of authors that has been remarkably linear over more than 20 years, without displaying the more typical phase of exponential growth. Nevertheless, our model provides an excellent fit to the data, even though parameter estimates force the numbers of exposed and adopters to their fixed points, as functions of a growing population $N$, at a relative growth rate of $7 \%$ a year. As a result the model solution that best fits the data for inflation is particularly sensitive to the growth dynamics of the population of susceptibles, and less so to the magnitude of the contact rate, as this factors out in the fixed point solution for $I$. It would be interesting to validate these inferences, or seek good fit solutions in different regimes.

Second, Quantum Computing and Computation shows a particularly long incipient period, with very slow growth over more than twenty years, and a quick (exponential) rise starting in the late 1980s. We modeled these dynamics by allowing for susceptible population growth starting only in 1990, which gives an excellent fit to the data. We note, however, that models with several exposed classes and therefore potentially longer 
successive incubation times (see Section 5), or with time-varying contact rates, may also provide viable alternatives. As for the case of inflation, it would be interesting to obtain more detailed historical data that could guide such detailed modeling choices.

An important component of predicting the future course of a scientific field is assessments of the sensitivity of goodness of fit from model solutions to perturbations in parameters. Figures 2-7 show the changes in model best fits due to the doubling of the contact rate between susceptibles and adopters. More systematic approaches capable of quantifying exhaustively which parameters lead to the greatest sensitivity at a specific stage of the dynamics are also being pursued through the study of sensitivity equations, and will be presented elsewhere [10]. This type of study will enable potential optimal interventions by funding agencies and other organizations, analogous in spirit to those designed to halt the spread of infectious diseases, but with the opposite intention of increasing the pace of scientific discovery and the spread of ideas.

We note in closing that the type of modeling described here can be enlarged in several interesting directions. One direction involves improvements to the model itself. The length of the infectious period, recruitment rates, and perhaps even incubation times may be measurable directly from publication data, $\mathrm{PhD}$ theses records, and so on. Knowledge of their distributions would help greatly to constrain and improve models, as well as distinguish whether the growth of a field is the primary result of the recruitment of new susceptibles, or instead the consequence of the conversion of an already large susceptible population via a larger contact rate. Additional features of the basic SEIR model may also be added, such as a model in which the size of the infected class facilitates further recruitment (directly linking $I$ with $\Lambda$ ). Such a model might account for the unusual pattern of publications on nanotechnology, which jumped discontinuously around 1990 [11]. Similarly, an explicit class of converts to competing ideas, $Z$ ("skeptics" or "stiflers"), may be added, as in [4]. This feature could prove especially useful for the combined case of inflation and cosmic strings, since many authors published on both topics over time.

Second, measures of scientific productivity can be produced by considering numbers of papers and authors over time, to judge if fields show increasing returns in scientific productivity resulting from the addition of new authors (increasing returns to scale) or otherwise. We believe that such studies will indicate whether a field shows vitality, in the sense of expanding opportunities per individual researcher, and that this feature may be a determinant of their future development and capacity to recruit. Related to this, studies that track trends in $\mathrm{PhD}$ theses in addition publications should help elucidate the roles played by institutions in the dynamics of scientific fields. For example, does the evolution in recruitment rates or susceptible populations derive mostly from having large numbers of students emerge from a few influential departments, or from having many departments train a smaller average number of practitioners? We have begun work on one such case, involving dissertations on superstring theory between the mid-1970s and today [12]. Work on this and related cases in the future may help us expand beyond simple measures like $R_{0}$ to quantify growth and change. 
Finally, detailed networks of authors, or of publications, can be built to quantify how relationships of collaboration, citation, or concept flow are structured and evolve over time. Such models yield the greatest level of detail, showing the specific paths and associations of individual authors or papers, and potentially complementing issues raised by epidemic models, which deal primarily with average properties of entire scientific communities.

\section{References}

[1] Derek J. de Solla Price. Little Science, Big Science (New York: Columbia University Press, 1963).

[2] E. Garfield. "The epidemiology of knowledge and the spread of scientific information," Current Contents 35, 5-10 (1980).

[3] W. Goffman and G. Harmon. "Mathematical approach to the prediction of scientific discovery," Nature 229, 103-104 (1971).

[4] L. M. A. Bettencourt, A. Cintron-Arias, D. I. Kaiser and C. Castillo-Chávez (2006a). "The power of a good idea: Quantitative modeling of the spread of ideas from epidemiological models," Physica A 364, 513-536 (2006).

[5] M. E. J. Newman. "Scientific collaboration networks: I. Network construction and fundamental results," Phys. Rev. E 64, art. no. 016131 (2001).

[6] M. E. J. Newman. "Scientific collaboration networks: II. Shortest paths, weighted networks, and centrality," Phys. Rev. E 64, art. no. 016132 (2001).

[7] M. E. J. Newman. "The structure of scientific collaboration networks," Proc. Natl. Acad. Sci. USA 98, 404-409 (2001).

[8] F. Brauer and C. Castillo-Chávez (2001). Mathematical models in population biology and epidemiology. Texts in Applied Mathematics, 40. (New York: Springer-Verlag, 2001).

[9] Alan Guth and David Kaiser. "Inflationary cosmology: Exploring the universe from the smallest to the largest scales," Science 307, 884-890 (2005).

[10] L. M. A. Bettencourt, A. Cintron-Arias, D. I. Kaiser and C. Castillo-Chávez. "On the spread of scientific knowledge," in preparation.

[11] David Kaiser. "Notes toward a nanotech timeline." DOE/OSTI technical report (2006).

[12] David Kaiser. "The ties that bind: Training string theorists in the United States, 1975-2005." DOE/OSTI technical report (2006). 
[13] John M. Barry. The Great Influenza: the epic story of the deadliest plague in history. Rev. ed. (New York: Penguin, 2005).

[14] G. Gensini, M. Yacoub, and A. Conti. "The concept of quarantine in history: from plague to SARS." Journal of Infection 49, no. 4, 257-61 (2004).

[15] Herbert W. Hethcote. "The mathematics of infectious diseases." SIAM Rev. 42, no. 4, 599-653 (2000).

[16] N. W. McLachlan. Modern operational calculus with applications in technical mathematics. Revised edition (New York: Dover, 1962).

[17] Horst R. Thieme. Mathematics in population biology. Princeton Series in Theoretical and Computational Biology. (Princeton, NJ: Princeton University Press, 2003).

[18] Horst R. Thieme. "The transition through stages with arbitrary length distributions, and applications in epidemics," in Mathematical approaches for emerging and reemerging infectious diseases: models, methods, and theory (Minneapolis, MN, 1999), 45-84, IMA Vol. Math. Appl., 126, Springer, New York, 2002.

[19] M. Fiedler and V. Ptak. "On matrices with positive non-positive off-diagonal elements and positive principal minors." Czechoslovak Math. J. 12, 382-400 (1962).

[20] A. Berman and R.J. Plemmons Nonnegative Matrices in the Mathematical Sciences. (New York: Academic Press, 1979).

[21] G. Plloe and T.Boullion. "A survey on M-matrices." SIAM Rev. 16, 419-427 (1974).

[22] J.M. Hyman and J. Li. "The reproductive number for an HIV model with differtial infectivity and staged progression." Linear Algebra and its Applications, 398, 101-116 (2005). 


\section{Appendix}

\section{Mathematical Extensions}

To study the dynamics of the transmission of an scientific idea, we formulate a model, following the approaches in mathematical epidemiology $([8,15,17])$ described by the following system of differential equations

$$
\begin{aligned}
& S^{\prime}(t)=\Lambda-\beta S \frac{I}{N}-b S \frac{Z}{N}-\mu S, \\
& E_{1}^{\prime}(t)=(1-p) \beta S \frac{I}{N}+(1-l) b S \frac{Z}{N}-\rho_{1} E_{1} \frac{I}{N}-(\mu+\gamma) E_{1}, \\
& E_{2}^{\prime}(t)=\gamma E_{1}-(\mu+\gamma) E_{2}-\rho_{2} E_{2} \frac{I}{N}, \\
& I^{\prime}(t)=p \beta S \frac{I}{N}+\rho_{1} E_{1} \frac{I}{N}+\gamma E_{2}+\rho_{2} E_{2} \frac{I}{N}-\mu I, \\
& Z^{\prime}(t)=l b S \frac{Z}{N}-\mu Z .
\end{aligned}
$$

Fig.8 is the transfer diagram for System 2 .

Linearize System 2 at $S \approx N$, and $E_{1} \approx 0, E_{2} \approx 0, I \approx 0, Z \approx 0$, we have

$$
\begin{aligned}
& E_{1}^{\prime}(t) \approx(1-p) \beta I+(1-l) b Z-(\mu+\gamma) E_{1}, \\
& E_{2}^{\prime}(t)=\gamma E_{1}-(\mu+\gamma) E_{2}, \\
& I^{\prime}(t)=p \beta I+\gamma E_{2}-\mu I, \\
& Z^{\prime}(t)=l b Z-\mu Z .
\end{aligned}
$$

Trivial steady-state is locally asymptotically stable if and only if

$$
\max \left(R_{0}^{I}, R_{0}^{Z}\right)<1
$$

where

$$
R_{0}^{Z}=\frac{l b}{\mu}
$$

is on average the secondary "stifler" individuals generated by one typical "stifler" individual in an otherwise susceptible population;

$$
R_{0}^{I}=\frac{p \beta}{\mu}+(1-p)\left(\frac{\beta}{\mu}\right)\left(\frac{\gamma}{\mu+\gamma}\right)\left(\frac{\gamma}{\mu+\gamma}\right)
$$

is on average the secondary "infectious" individuals generated by one typical "infectious" individual in an otherwise susceptible population. The first term in $R_{0}^{I}$ is the contribution of transmission from $S$ to $I$ directly while the second term corresponds to the contribution of transmission from $S$ to $I$ through $E_{1}$ and $E_{2}$. 


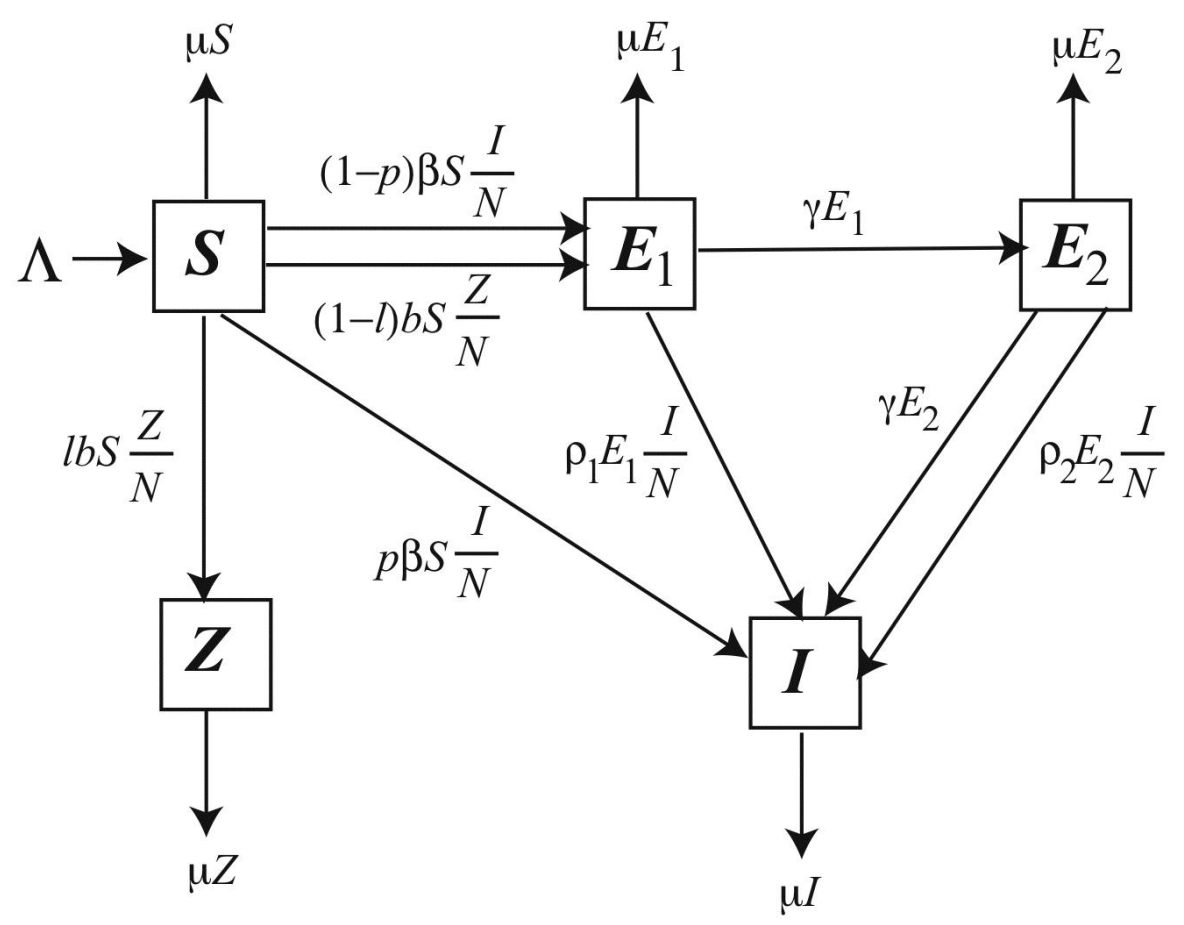

Figure 8: Transfer diagram for the SEIZ model.

\section{Model with $n$ Exposed Classes}

We consider a situation when people get exposed to an scientific idea, there are $n$ learning stages before they fully understand the idea and be able to teach ("infect") others. Let $E_{i}$ denote the $i-t h$ class of exposed individuals for $i=1,2, \cdots, n$. Then, following similar modeling approaches as those used in epidemiology $([8,15,17])$, we arrive the following model 


$$
\begin{aligned}
& S^{\prime}(t)=\Lambda-\beta S \frac{I}{N}-b S \frac{Z}{N}-\mu S \\
& E_{1}^{\prime}(t)=(1-p) \beta S \frac{I}{N}+(1-l) b S \frac{Z}{N}-\rho_{1} E_{1} \frac{I}{N}-(\mu+\gamma) E_{1}, \\
& E_{2}^{\prime}(t)=\gamma E_{1}-(\mu+\gamma) E_{2}-\rho_{2} E_{2} \frac{I}{N} \\
& E_{3}^{\prime}(t)=\gamma E_{2}-(\mu+\gamma) E_{3}-\rho_{3} E_{3} \frac{I}{N} \\
& \cdots \\
& E_{n}^{\prime}(t)=\gamma E_{n-1}-(\mu+\gamma) E_{n}-\rho_{n} E_{n} \frac{I}{N}, \\
& I^{\prime}(t)=p \beta S \frac{I}{N}+\rho_{1} E_{1} \frac{I}{N}+\rho_{2} E_{2} \frac{I}{N}+\rho_{3} E_{3} \frac{I}{N}+\cdots+\gamma E_{n}+\rho_{n} E_{n} \frac{I}{N}-\mu I, \\
& Z^{\prime}(t)=l b S \frac{Z}{N}-\mu Z .
\end{aligned}
$$

The system diagram is shown in Fig.9. Similar to the case where we have two exposed classes, we can find the basic reproductive numbers $R_{0}^{Z}$ and $R_{0}^{I}$ for "stifler" and infectious classes respectively as given in Eq.7 and Eq.8.

$$
\begin{gathered}
R_{0}^{Z}=\frac{p \beta}{\mu} \\
R_{0}^{I}=(1-p)\left(\frac{\beta}{\mu}\right)\left(\frac{\gamma}{\mu+\gamma}\right)^{n},
\end{gathered}
$$

and define $R_{0}=\max \left\{R_{0}^{Z}, R_{0}^{I}\right\}$.

By using the same approach as J.M. Hayman and J. Li used [22], we can show that the trivial steady-state is locally asymptotically stable if $R_{0}<1$, and it is unstable if $R_{0}>1$. In fact, the Jacobian matrix of system(6) at the trivial steady-state is given by

$$
J_{1}=\left[\begin{array}{ccccccc}
-(\mu+\gamma) & 0 & 0 & \cdots & 0 & (1-p) \beta & (1-l) b \\
\gamma & -(\mu+\gamma) & 0 & \cdots & 0 & 0 & 0 \\
0 & \gamma & -(\mu+\gamma) & \cdots & 0 & 0 & 0 \\
\cdots & & 0 & \cdots & -(\mu+\gamma) & 0 & 0 \\
0 & 0 & 0 & \cdots & \gamma & p \beta-\mu & 0 \\
0 & 0 & 0 & \cdots & 0 & 0 & l b-\mu
\end{array}\right]
$$

From the last row of $J_{1}$, we can see that $J_{1}$ has an eigenvalue $l b-\mu$, which is negative when $R_{0}=\max \left\{R_{0}^{Z}, R_{0}^{I}\right\}<1$. 
Define

$$
J=\left[\begin{array}{cccccc}
-(\mu+\gamma) & 0 & 0 & \cdots & 0 & (1-p) \beta \\
\gamma & -(\mu+\gamma) & 0 & \cdots & 0 & 0 \\
0 & \gamma & -(\mu+\gamma) & \cdots & 0 & 0 \\
\cdots & & & & & \\
0 & 0 & 0 & \cdots & -(\mu+\gamma) & 0 \\
0 & 0 & 0 & \cdots & \gamma & p \beta-\mu
\end{array}\right]
$$

Recall from well known M-matrix theory that if $B=\left[b_{i j}\right]$ is an irreducible $n \times n$ matrix with $b_{i i} \geq 0$, and $b_{i j} \leq 0$, for $i \neq j, i, j=1, \cdots, n$, then the real part of every nonzero eigenvalue of $B$ is positive if and only if there exists a positive vector $x>0$ such that $B x \geq 0[19,20,21]$.

Let $B=-J$, since $0<p<1$, it is easy to see that $\mathrm{B}$ is irreducible, the diagonal elements of $B$ are positive and the off-diagonal elements of $B$ are non-positive. Define the following vector

$v=\left(\frac{\mu-p \beta}{\mu(\mu+\gamma)}, \frac{\mu-p \beta}{\mu(\mu+\gamma)}\left(\frac{\gamma}{\mu+\gamma}\right), \frac{\mu-p \beta}{\mu(\mu+\gamma)}\left(\frac{\gamma}{\mu+\gamma}\right)^{2}, \cdots, \frac{\mu-p \beta}{\mu(\mu+\gamma)}\left(\frac{\gamma}{\mu+\gamma}\right)^{n-1}, \frac{1}{\mu}\left(\frac{\gamma}{\mu+\gamma}\right)^{n}\right)^{T}$,

then $v$ is a positive vector if $R_{0}<1$. Since when $R_{0}<1$

$$
B \cdot v=\left(1-R_{0}, 0,0, \cdots, 0,0\right)^{T} \geq 0,
$$

according to the M-matrix theory, every nonzero eigenvalue of $B$ has positive real part. Hence, every nonzero eigenvalue of $J$ has negative real part. Finally, calculating the deter- 
minant of $J$, we get

$$
\begin{aligned}
& |J|=\left|\begin{array}{cccccc}
-(\mu+\gamma) & 0 & 0 & \cdots & 0 & (1-p) \beta \\
\gamma & -(\mu+\gamma) & 0 & \cdots & 0 & 0 \\
0 & \gamma & -(\mu+\gamma) & \cdots & 0 & 0 \\
\cdots & & & & & \\
0 & 0 & 0 & \cdots & -(\mu+\gamma) & 0 \\
0 & 0 & 0 & \cdots & \gamma & p \beta-\mu
\end{array}\right| \\
& =-(\mu+\gamma)\left|\begin{array}{cccccc}
-(\mu+\gamma) & 0 & 0 & \cdots & 0 & 0 \\
\gamma & -(\mu+\gamma) & 0 & \cdots & 0 & 0 \\
0 & \gamma & -(\mu+\gamma) & \cdots & 0 & 0 \\
\cdots & & & & & \\
0 & 0 & 0 & \cdots & -(\mu+\gamma) & 0 \\
0 & 0 & 0 & \cdots & \gamma & p \beta-\mu
\end{array}\right| \\
& +(-1)^{n}(1-p) \beta\left|\begin{array}{cccccc}
\gamma & -(\mu+\gamma) & 0 & \cdots & 0 & 0 \\
0 & \gamma & -(\mu+\gamma) & \cdots & 0 & 0 \\
0 & 0 & \gamma & \cdots & 0 & 0 \\
\cdots & & & & & \\
0 & 0 & 0 & \cdots & \gamma & -(\mu+\gamma) \\
0 & 0 & 0 & \cdots & 0 & \gamma
\end{array}\right| \\
& =(-1)^{n}(\mu+\gamma)^{n}(p \beta-\mu)+(-1)^{n}(1-p) \beta \gamma^{n} \\
& =(-1)^{n}\left(R_{0}-1\right) \mu(\mu+\gamma)^{n} \neq 0 \text { if } R_{0}<1 \text {. }
\end{aligned}
$$

that means, $J$ has no zero eigenvalue.

Therefore, when $R_{0}<1$, each eigenvalue of $J_{1}$ has negative real part, hence, the trivial steady-state is locally asymptotically stable.

Now consider $R_{0}>1$. If $R_{0}^{Z}>1$, then $J_{1}$ has a positive eigenvalue $l b-\mu$. If $R_{0}^{I}>1$, when $n$ is odd, we have $\left|J_{(n+1) \times(n+1)}\right|<0$, i.e., the product of even number of eigenvalues of $J$ is negative, so $J$ must have a positive eigenvalue; when $n$ is even, $\left|J_{(n+1) \times(n+1)}\right|>0$, i.e., the product of odd number of eigenvalues of $J$ is positive which again implies that $J$ has a positive eigenvalue. Therefore, when $R_{0}>1, J_{1}$ always has a positive eigenvalue, hence, the trivial steady-state is unstable. 


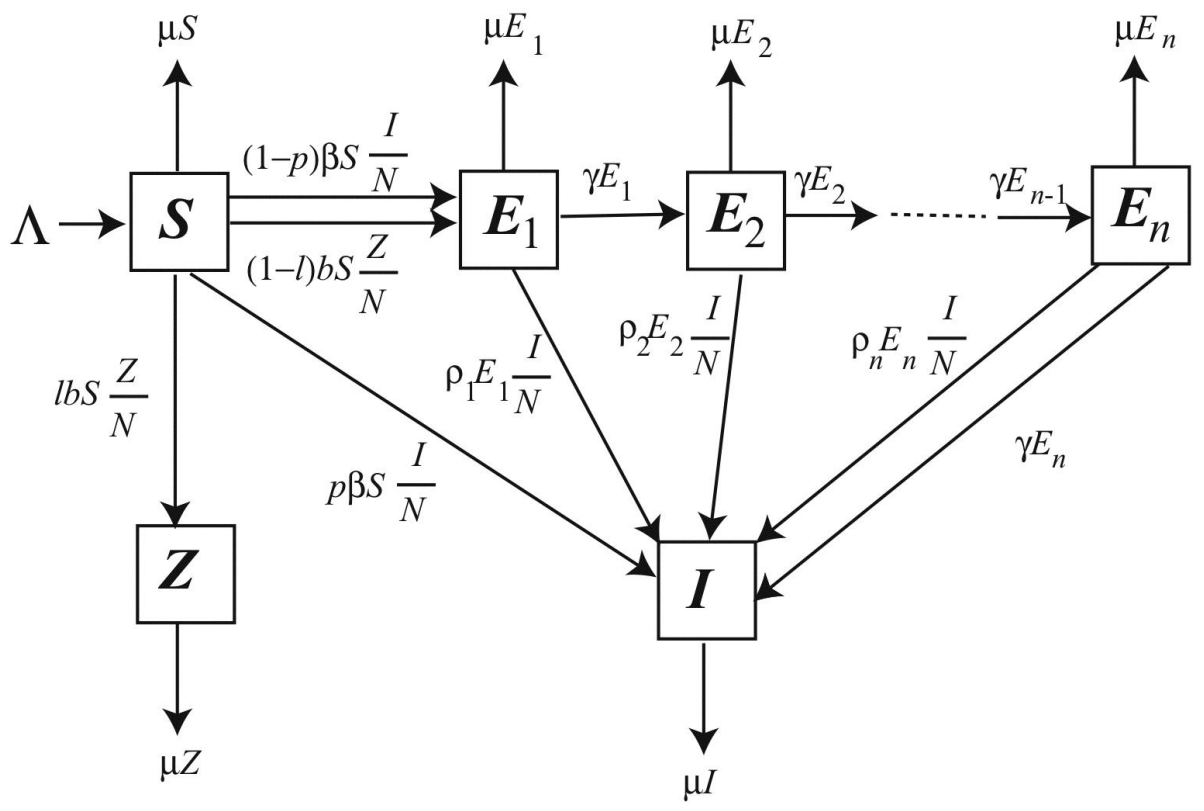

Figure 9: Transfer diagram for the SEIZ model with $n$ exposed classes. 